\title{
TEXTSORTE ALS NATÜRLICHKEITSTHEORETISCHE ENTITÄT
}

\section{Vorbemerkungen}

Die Produktion von Texten und Diskursen zählt zweifelsohne zu den komplexesten geistigen Aktivitäten des Menschen, die ihm durch das Interagieren der kognitiven Systeme (Erkennung von sprachlichen Mustern) mit jenen Systemen, die für die Sprachproduktion zuständig sind, ein unter Einhaltung gewisser Voraussetzungen äusserst effizientes Kommunizieren mit seiner Umwelt ermöglicht. Ein derart komplexes (sprachliches) Handeln kann nur dann erfolgreich sein, wenn es von Prinzipien der regelgeleiteten Abhängigkeit der sprachlichen / textuellen Elemente und einer höheren, sagen wir natürlichen Ordnung geleitet wird. In diesem Zusammenhang sei auf die Tatsache verwiesen, dass auch die als »Ausnahmen« bekannten grammatischen Erscheinungen, wo es zu untypischen oder nichtoptimalen Kodierungen kommt, regelgeleitet sind (Siehe hierzu auch Leiss 1992). (Beispiel: die Verwendung des Präsens zum Ausdruck anderer Tempora). Die Regelgeleitetheit der Sprache muss per definitionem auf allen Ebenen der Sprach- bzw. Textproduktion beobachtbar sein. Und wenn darüberhinaus allgemein auch die Interdependenz und die Interaktivität klar erkennbare, universell geltende Entitäten der Sprache sind, kann daraus das Vorhaben der vorliegenden Ausführungen abgeleitet werden. So wird im folgenden versucht, auf die Wirkung der Textsorte oder genauer des Textsortenwechsels auf das grammatische Mikroniveu einzugehen.

Berücksichtigt werden zwei Textexemplare, die jeweils zwei verschiedenen Texttypen zugeordnet werden können. Nach Diewald 1995 entspricht das erste repräsentativ ausgewählte Textexemplar der Grundtextsorte II (Diewald 1995 : 29) es handelt sich um das Telefongespräch, wogegen sich das zweite, die Talkshow, zumindest nach dem Grad der Komplexität, sowie der Ort- und Zeitgleichheit noch am ehesten der Grundtextsorte IV (Diewald, 1995 : 30) annähert. Im konkreten Fall handelt es sich beim zweitgenannten Textexemplar eigentlich um eine Subvariante: dem öffentlichen Dialog unter mehreren Beteiligten, die auch mit dem von Müller 1995 angeführten Redekonstellationstyp IV in gewisser Hinsicht korrespondiert. In der weiteren Folge wird jedoch der Überschaubarkeit willen terminologisch lediglich auf zwei verschiedene Textsorten Bezug genommen, da hier die nach wie vor lebhafte Polemik über die Kategorisierung von Texten bzw. Textvorkommen nicht ausführlich 
diskutiert werden kann. Es sei demnach festgehalten, dass beide Textsorten durch unterschiedlich ausgeprägte Markiertheitsgrade des dialogischen Prinzips gekennzeichnet werden. Innerhalb des erwähnten Textsortenpaares wird dem Telefongespräch ein geringerer Markiertheitsgrad beigemessen (auch bei Diewald 1995 : 29 wird das Telefongespräch als »die unmarkierte Ausprägung des dialogischen Prinzips « eingeordnet), was als Konsequenz durch unmittelbare Reaktion auf das Mikroniveau der jeweiligen grammatischen/pragmatischen Dependenzbeziehungen beobachtbar sein müsste.

Bevor jedoch in diesem Zusammenhang auf die Einzelheiten näher eingegangen werden kann, soll die Betrachtungsperspektive des slowenischen Modells der NATÜRLICHEN SYNTAX vorgeführt und in Relation zur Textsorte beleuchtet werden.

\section{II. Über die NATÜRLICHE SYNTAX}

Die Beschreibungs- und Erklärungsstrategie der sprachlichen Phänomene wird von der Natürlichen Syntax unter Inanspruchnahme folgender Natürlichkeitsprinzipien vorgenommen: Ikonizität/Kontraikonizität, Segmentierungsikonismus, Systemangemessenheit, Erwartbarkeit (Präsupposition), Markiertheitsverhältnisse, Token-Frequenz, Anzahl und Komplexität der Generierungsoperationen und Spracherwerbsabfolge (Siehe hierzu auch Wurzel 1992, Orešnik et al. 1990, Mayerthaler 1993, 1995, Dressler 1985, Leiss 1992, Teržan-Kopecky 1995). Über die Notwendigkeit, diese Kriterien auch noch durch die TEXSORTENSENSIBILITÄT zu ergänzen, die sowohl auf dem sprachlichen Makroniveau als auch auf dem Mikroniveau als ein äußerst wirksames Kriterium der Natürlichkeit und zugleich als Kriterium zur Bestimmung von Abgrenzungen etwa zwischen Texttypen und Textsorten dementsprechend ernst zu nehmen ist, wird in der weiteren Folge, auch unter Einbeziehung einer empirischen Pilotstudie, die sich mit pragmatischen Parametern auseinandersetzt, näher eingegangen.

An den o.a. grammatischen/sprachlichen Entitäten werden die Natürlichkeitsgrade und die Grammatikalisierungsphasen gemessen sowie die kategorialen Umrisse (= ihre unstabilen Grenzen) festgelegt. Das von mir aufgestellte natürlichkeitstheoretisch orientierte Funktionsmodell schlägt eine spezifische Ausprägung der natürlichen Merkmale der syntaktischen Kategorien, auch unter Berücksichtigung der Variable Textsorte, vor. 


\section{III. Über die Distributionseigenschaften der syntaktischen Kategorien:}

3.1. Die grammatischen Eigenschaften von syntaktischen Konstruktionen werden durch typische/charakteristische Ausprägungen ausgewiesen, was bedeutet, dass für jede syntaktische Konstruktion eine typische Distributionswahrscheinlichkeit in ihrer unmittelbaren grammatischen Umgebung vorausgesetzt wird. Die typische Distributionswahrscheinlichkeit von sogenannten verstärkteren syntaktischen Konstruktionen ist ihr im Vergleich mit ihren schwächeren syntaktischen Varianten intensiveres Kollozieren mit markierteren Varianten der grammatischen Parameter, die ihr Auftreten im Text unmittelbar begleiten.

(1) Das Perfekt kolloziert als syntaktische Verstärkung im Deutschen im Vergleich zum schwächeren Präteritum intensiver mit markierteren Varianten der berücksichtigten grammatischen Parameter. Die Beobachtung wurde auf allen untersuchten Entfaltungsstufen (stichprobenweise untersuchte literarische Texte aus dem 13., 15., 16. und 20. Jahrhundert) gemacht. Der Unterschied in der Markiertheit beider Konstruktionen liegt im 13. und im 20. Jahrhundert sehr niedrig (bei 34\%), weil es sich im ersten Fall um die Vorgrammatikalisierungsstufe des Perfekts handelt, im zweiten Fall aber um seine Postgrammatikalisierungsphase, wo sich eine Ausgleichstendenz mit der schwächeren Variante (= dem Präteritum) bemerkbar macht. Die höchste (hypothesenfreundliche) Abweichung zu Gunsten der Markiertheit des Perfekts wurde im Text aus dem 15. Jahrhundert verzeichnet. Sie betrug $83 \%$. Ein solcher Zustand markiert die Grammatikalisierungsphase einer Konstruktion.

(2) Das worden-Passiv kolloziert als verstärkte Konstruktion im Vergleich mit dem Durchschnitt aller anderen Konstruktionen im Text aus dem 17. Jahrhundert um ganze 75\% intensiver mit markierteren Parametervarianten.

3.2. Das prototypische grammatische Umfeld von verstärkten syntaktischen Konstruktionen bilden demnach grammatische Parameter, die von hohen Markiertheitsstufen gekennzeichnet werden.

3.3. Das Auftreten von syntaktischen Konstruktionen in ihrer oben explizierten prototypischen grammatischen Umgebung wird als Idealfall der Realisierung der Natürlichkeitsprinzipien in einer Sprache verstanden. Ein solcher Idealfall ist jedoch nur dann gegeben, wenn eine gewisse syntaktische Konstruktion in einer Textsorte auftritt, welche für sie am natürlichsten ist. Als konkrete Folge dieses Natürlichkeitsprinzips kann z. B. die Tatsache interpretiert werden, dass das Präteritum im Deutschen in der Textsorte Roman die höchste Distributionsfrequenz aufweist, daher wird es in einer solchen kontextuellen Umwelt von weniger markierten Parametervarianten begleitet. Das gesprochene Gegenwartsdeutsch stellt aber für diese Konstruktion eine weniger natürliche Textsorte dar. Durch diesen Umstand wird eine natürliche Reaktion der Sprache ausgelöst, wonach der Markiertheitsgrad der kollozierenden grammatischen Parameter stark verstärkt wird.

(3) Für das Präteritum ist die Umgangssprache eine michtprototypische Textsorte, daher lässt es in solchen Umgebungen viel weniger der für es umproduktiven perfektiven Verben zu. In 
den bearbeiteten Texten (Talkshows) um 20\% weniger als in prototypischen Textsorten (etwa Roman).

(4) Für das Perfekt ist der Roman eine nichtprototypische Textsorte, daher lässt es in solchen Kontexten viel weniger der für es unproduktiven imperfektiven Verben $\mathrm{zu}$. (Im bearbeiteten Sprachmaterial um $20 \%$ weniger)

(5) Die Markiertheitsstufen vom analytischen Futur vom Typ werde veranlassen und vom Präsens pro futuro vom Typ bald regnet es weisen in der Textsorte Roman im 20. Jahrhundert kaum Unterschiede auf. Der Unterschied in der Markiertheitsdichte der kollozierenden grammatischen Parameter des analytischen Futurs übersteigt aber in einer anderen Textsorte dem gesprochenen Gegenwartsdeutsch um ganze 63\% die Markiertheit des Präsens pro futuro.

(6) Die Belege des Präsens pro futuro sind in der für sie prototypischen Textsorte - der modernen deutschen Sprechsprache (in der bereits explizierten Definition der Markiertheit ihres unmittelbaren grammatischen Umfeldes) um 14\% weniger markiert als in den anderen bearbeiteten Textsorten aus dem 20. Jahrhundert. Sie sind jedoch global betrachtet in allen Textsorten stabiler als die Belege des analytischen Futurs, die ihrerseits bereits über Jahrhunderte hindurch starken Umkategorisierungsprozessen ausgesetzt sind.

3.8. Das oben Dargelegte veranschaulicht den Umstand, dass die Markiertheitsstufen von syntaktischen Konstruktionen im positiven Verhältnis zur Natürlichkeit bzw. zur Prototypizität DER TEXTSORTE stehen.

3.9. Eine neue syntaktische Konstruktion wird auch nach der erfolgten Grammatikalisierung in ihrer prototypischen Textsorte am ehesten transparent, wodurch hohe Markiertheitsstufen redundant werden. Eine solche textsortenfreundliche Distribution entspricht auch den Erwartungshaltungen der Rezipienten und kann als präsupponiert gelten. Eine Ausnahme ist in diesem Zusammenhang erneut das Auftreten in nichtprototypischen Textsorten .

3.10. In der Sprache kann man jedoch sehr oft auch so genannte kontraikonische, das heisst, den Erwartungshaltungen der Rezipienten nicht entsprechende Kodierungen beobachten. Solche Kodierungsstrategien werden von sprachlichen Ökonomieprinzipien geleitet. Danach besteht eine innersprachliche Tendenz, die Beziehungen unter den sprachlichen Elementen derart zu regeln, dass ein Minimum an Energie in die Sprachproduktion investiert werden muss. Ein solches sprachliches Verfahren ist jedoch nur dann zulässig, wenn dadurch die Effizienz des Informations- bzw. Intentionstransfers nicht beeinträchtigt wird.

3.6.1. Auch zu kontraikonischen Kodierungen kommt es am ehesten in jener Textsorte, die für die jeweilige Konstruktion weniger natürlich bzw. nichtprototypisch ist.

(7) Das analytische Futur wird vom kontraikonischen Präsens pro futuro in der für es nichtprototypischen Textsorte gesprochenes Gegenwartsdeutsch am massivsten verdrängt. 


\section{DAS VERHÄLTNIS TEXTSORTE - MARKIERTHEIT DER SYNTAKTISCHEN KONSTRUKTIONEN AUS PRAGMATISCHER SICHT}

Um das oben Vorgeführte auch aus pragmatischer Sicht auszuleuchten, wurde eine Pilotstudie vorgenommen, die in der weiteren Folge näher vorgestellt und diskutiert werden soll.

\subsection{Methode:}

4.1.1. Untersucht wurden zwei Textsorten: Das Telefongespräch und die Talkshow. Die Letztgenannte wurde als repräsentativ für die als öffentlicher Dialog klassiffizierbare Textsorte gewählt, wobei im konkreten Fall jeweils zwei Personen vor einem Publikum miteinander kommunizieren. Für eine solche Kommunikationssituation wurde angenommen, dass durch die Steigerung des Formalitäts- bzw. Öffentlichkeitsgrades, durch die Vielzahl der potentiellen Dialogpartner und Zuhörer, sowie durch die Themafixierung und den argumentativen Charakter eine Präferenz zu markierteren grammatischen/pragmatischen Parametern erwartbar ist. Es wurde die Vorkommenshäufigkeit folgender Parameter in beiden Textsorten statistisch ausgewertet: Länge der Replik, Länge einzelner Sätze, Komplexität der Konjunktionen, Komplexität der Kasus- und Tempusformen, Substantiv gegenüber Proform, Abgeschlossenheit gegenüber Nichtabgeschlossenheit von Sätzen sowie die Opposition Realis/Irrealis.

4.1.2. Als hypothesenfreundliche, positive Ereignisse galten größere Distributionsdichten markierterer Parametervarianten in der komplexeren Textsorte Talkshow als im weniger komplexen Telefongespräch. Folgende Parametervarianten wurden als markiertere und somit im Falle ihrer Distribution in der komplexeren Textsorte günstige Auftretensmodalitäten klassiffiziert: eine mehr als zwei Sätze enthaltende Replik; aus sieben oder mehr als sieben Elementen bestehende Sätze; Auftreten von analytischen Konjunktionen vom Typ: und dann; Auftreten von analytischen Kasusformen; relative Verbaltempora; weniger Proformen; mehr abgeschlossene als nichtabgeschlossene Sätze und Irrealis.

\subsection{Statistische Angaben}

4.2.1. lange Replik:

4.2.2. lange Sätze:

4.2.3. komplexe Konjunktionen:

$1=$ Textsorte Telefongespräch

$2=$ Textsorte Talkshow

$$
\begin{array}{ll}
\mathrm{P}(\mathrm{a} 1)^{1}=\mathbf{3 0 \%} & \mathrm{P}(\mathrm{a} 1 \mathrm{~b})^{2}=\mathbf{7 5 \%} \\
\mathrm{P}(\mathrm{a} 2)=\mathbf{2 3 \%} & \mathrm{P}(\mathrm{a} 2 \mathrm{~b})=\mathbf{3 5 \%} \\
\mathrm{P}(\mathrm{a} 3)=\mathbf{3 \%} & \mathrm{P}(\mathrm{a} 3 \mathrm{~b})=\mathbf{1 5 \%}
\end{array}
$$


4.2.4. analytische Kasusformen:

$$
\begin{array}{ll}
\mathrm{P}(\mathrm{a} 4)=\mathbf{1 7 \%} & \mathrm{P}(\mathrm{a} 4 \mathrm{~b})=\mathbf{3 0 \%} \\
\mathrm{P}(\mathrm{a} 5)=\mathbf{3 \%} & \mathrm{P}(\mathrm{a} 5 \mathrm{~b})=\mathbf{9 \%} \\
\mathrm{P}(\mathrm{a} 6)=\mathbf{8 3 \%} & \mathrm{P}(\mathrm{a} 6 \mathrm{~b})=\mathbf{8 7 \%} \\
\mathrm{P}(\mathrm{a} 7)=\mathbf{7 5 \%} & \mathrm{P}(\mathrm{a} 7 \mathrm{~b})=\mathbf{7 7 \%} \\
\mathrm{P}(\mathrm{a} 8)=\mathbf{2 \%} & \mathrm{P}(\mathrm{a} 8 \mathrm{~b})=\mathbf{4 \%}
\end{array}
$$

4.2.5. relative Tempora:

4.2.6. keine Proformen :

4.2.7. abgeschlossene Sätze:

4.2.8. Irrealis:

Markiertheit von pragmatischen Parametern in der Textsorte Telefongespräch und Talkshow

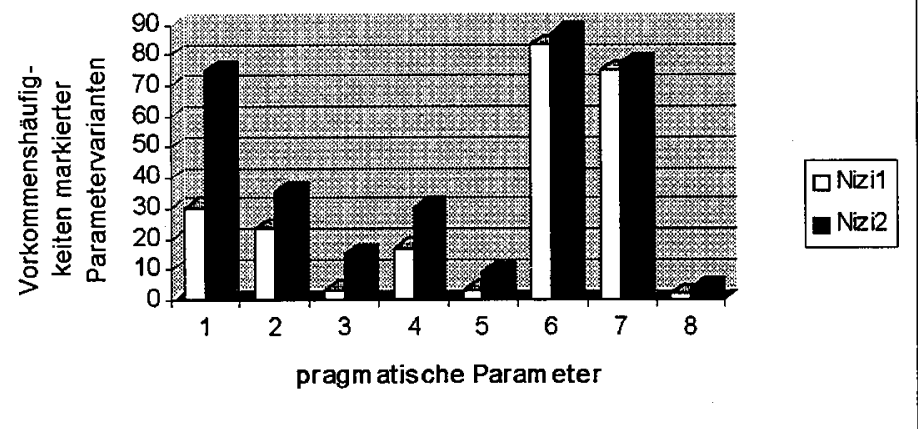

\section{Erläuterungen zu den gewonnenen Ergebnissen der} Textanalyse

5.6. Angesichts der Eindeutigkeit der statistischen Ergebnisse, die zu 100\%, d.h. bei allen in die statistische Datenauswertung aufgenommenen pragmatischen Parametern die aufgestellte Hypothese bestätigen, bleibt ihre eigene Aussagekraft lediglich dahingehend zu untermauern, dass diese Werte sicherlich nicht dem Zufall der begrenzten Menge des repräsentativ ausgewählten Korpuses zuzuschreiben ist. Die Komplexitätsskala beider Samples ist nämlich, obwohl sie verschiedene Textsorten repräsentieren, sehr ähnlich strukturiert. Den angeführten Daten kann man entnehmen, dass die Abweichungen zwischen den ausgewiesenen Prozentwerten nicht derart drastisch sind, als dass sie keine parameterbedingten Präferenzen verdeutlichen würden. In diesem Sinne ist auch eine textsortenunabhängige Invarianz in den Markiertheitsbeziehungen unter den Parametern selbst zu beobachten. Sie besitzen nämlich auch universelle Distributionspräferenzen. Demzufolge erwies sich Irrealis unabhängig von der Textsorte als der markierteste Parameter, gefolgt von den relativen Tempora, der Komplexität von Konjunktionen und der Abgeschlossenheit von Sätzen. Für Parameter solcher Art werden in allen Textsorten niedrige Distributionswerte vorausgesetzt, die niedrigsten wohl in weniger markierten Textsorten. 
Parameter wie Länge von Sätzen und Repliken, sowie Auftreten von analytischen Konstruktionen sind hingegen stark textsortenbezogen und weisen daher auch in unserem Fall die stärksten Abweichungen aus, da sie ja selber stark situationsgebunden sind. Indem sie von außersprachlichen Leistungen / Gegebenheiten massiv Gebrauch machen und äußerst wirkungsorientiert sind, kann man sie als prototypische pragmatische Parameter bezeichnen.

\section{Abschließende Bemerkungen}

6.7. Das oben Dargelegte, eindeutig hypothesenfreundliche Ergebnis, rechtfertigt die Relevanz des Textsortenwechsels für die Distribution der grammatischen bzw. pragmatischen Werte. Dabei gilt der natürlichkeitstheoretische Grundsatz, wonach stark markierte bzw. verstärkte Parameter zwar in allen Textsorten selten sind, ihre Frequenz jedoch in markierteren Textsorten merklich steigt. Das Gegenteil gilt für schwächere Parameter. Diese Beobachtungen beanspruchen auch die Aussagekraft in Bezug auf die Abgrenzungsversuche von Texttyp - Textklasse - Textsorte, indem u.a. auch von den Markiertheitsverhältnissen auf dem grammatischen Mikroniveau bei der Klassifizierung von Textvorkommen ausgegangen werden kann. Konkret wurde von der eben besprochenen empirischen Textauswertung die Textsorte Talkshow gegenüber der Textsorte Telefongespräch von einer entsprechend höheren Markiertheitsstufe der beobachteten pragmatischen Parameter ausgewiesen. (In allen Parametern übersteigen die aus den Talkshows gewonnenen Werte jene, die in Telefongesprächen ausgewiesen wurden.)

6.8. Der Textsortenwechsel löst, um es nochmals festzuhalten, äußerst prägnante Reaktionen sowohl auf dem grammatischen als auch auf dem pragmatischen Mikroniveau aus. Diese Tatsache rechtfertigt die eingangs aufgesetzte Proposition, wonach die Textsortensensibilität als eines der natürlichkeitstheoretischen Kriterien gelten soll, an denen einerseits das gegenseitige Verhältnis von Konstruktionspaaren und andererseits das Verhältnis Texttyp/Textsorte im oben explizierten Sinn gemessen und abgegrenzt werden kann.

\section{Literatur}

Diewald, Gabriele: Textsortenklassifikation auf der Basis kommunikativer Grundbedingungen. In: Linguistica XXXV,1 Besedilne vrste - Textsorten. Ljubljana 1995. S. 21-36.

Dressler, Wolfgang Ulrich: Typological Aspects of Natural Morphology. In: Acta Linguistica Academiae Scientiarum Hungaricae. Budapest 1985. S. 51-70.

Leiss, Elisabeth: Die Verbalkategorien des Deutschen. Berlin 1992.

Mayerthaler, Willi: Morphologische Natürlichkeit. Wiesbaden 1991.

Müller, Rolf: Textsorten - natürliche Sprachformen oder kulturelle Sprachformen? In: Linguistica XXXV, 1 Besedilne vrste - Textsorten. Ljubljana 1995. S. 37-51. 
Orešnik, Janez/Snedec, Andrej/Teržan, Karmen/Trobev̌̌ek-Drobnak, Frančiska: I Introduction to the Subsequent Three Papers in the Present Volume. In: Linguistica XXX. Ljubljana 1990. S. 5-12.

Teržan-Kopecky, Karmen: Zu den Entfaltungseigenschaften der Verbalkategorien. In: Papiere zur Linguistik 53, 2. Tübingen 1995. S. 99-109.

Wurzel, Wolfgang Ullrich: Faktoren des Sprachwandels. In: Papiere zur Linguistik 45, 1/2. Tübingen 1992. S. 159-173.

\section{Zusammenfassung}

Die in den Textsorten Talkshow und gesprochenes Gegenwartsdeutsch vorgenommene empirische Auswertung der Kollokationstendenzen der beobachteten pragmatischen Parameter (Länge der Replik, Länge einzelner Sätze, Komplexität der Konjunktionen, Komplexität der Kasus- und Tempusformen, Substantiv gegenüber Proform, Abgeschlossenheit gegenüber Nichtabgeschlossenheit von Sätzen, Realis/Irrealis) wurde als Methode zur Festlegung von Texttypen- bzw. Textsortenabgrenzungen vorgeschlagen. Die Textsorte wurde auch als eines der wesentlichen natürlichkeitstheoretischen Entitäten eingestuft, da sich die festgestellten Markiertheitsunterschiede verschiedener Kategorien auf dem grammatischen Mikroniveau in eindeutiger Abhängigkeit zum Wechsel der Textsorte bewegen. Die aufgestellte Arbeitshypothese wurde zu $100 \%$ bestätigt. Auch in den vorangegangenen Untersuchungen $\mathrm{zu}$ den Distributionsmodalitäten der Tempora im Deutschen zeichneten sich ähnliche Präferenzen ab. Im Wesentlichen zeigt sich die auf die Textsortensensibilität bezogene natürliche Eigenschaft der grammatischen Kategorien in der Realisierung des Grundsatzes: in einer für eine gewisse Konstruktion natürlicheren textuellen Umwelt wird diese von weniger markierten grammatischen Parametern begleitet, als dies in einer für sie weniger natürlichen Textsorte der Fall ist.

Povzetek

BESEDILNA VRSTA KOT ENTITETA NARAVNEGA JEZIKOSLOVJA

\footnotetext{
Članek prikazuje empirično raziskavo teženj sopojavljanja opazovanih pragmatičnih parametrov (dolžina replike, dolžina posameznih stavkov, zapletenost veznikov, zapletenost sklonov in glagolskih oblik, samostalnik nasproti za-obliki, zaključenost nasproti nezaključenosti stavkov, realis/irealis) kot učinkovito metodo za opredeljevanje besedilnih vrst. Besedilna vrsta je opredeljena kot ena temeljnih vrednosti TEORIJE NARAVNOSTI. Ta postulat utemeljuje dejstvo, da so se ugotovljene stopnje zaznamovanosti kategorij na slovničnem mikronivoju gibale $\mathrm{v}$ jasno izpričani odvisnosti od besedilne vrste. Postavljena delovna domneva je bila $100 \%$-no potrjena. Podobne preference so pokazale tudi raziskave distribucijskih teženj glagolskih casov v nemšcini. Naravno lastnost slovničnih kategorij, ki jo odraža sprememba besedilne vrste, lahko $\mathrm{v}$ njenih temeljnih obrisih strnemo takole: $v$ besedilnem svetu, ki je za neko zgradbo najnaravnejši, spremljajo zgradbo manj zaznamovani slovnični parametri kot $v$ besedilni vrsti, ki je zanjo manj naravna.
} 